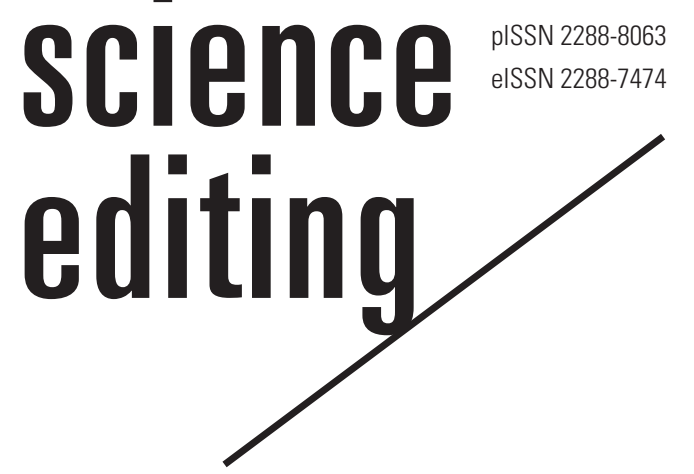

\title{
Increased number of papers co-authored by professor and his students in humanities and social sciences journals published in Korea
}

\author{
Rae Seong Hong ${ }^{1}$, Eun Seong Hwang ${ }^{2}$ \\ Departments of ${ }^{1}$ Korean Language and Literature, ${ }^{2}$ Life Science, University of Seoul, Seoul, Korea
}

\section{Abstract}

Humanities and social sciences studies in Korea have remarkably low rates of co-authorship between professors and students. We chose a bibliometrics-based approach to characterize changes in the ratio of joint authorship between professors and students. Articles classified in the humanities and social sciences sectors that were published in journals registered in the Korean Citation Index during 2 phases over a 10-year period-2004 to 2006 (phase 1) and 2011 to 2013 (phase 2)-were used as the main source for the analysis. The study results can be summarized as follows: first, the overall number of co-authored articles drastically increased from phase 1 to phase 2 ; the percentage of co-authorship articles increased from $34.8 \%$ to $47.7 \%$, and the percentage of co-authorship between students and professors rose from $9.9 \%$ to $20.7 \%$. This trend was particularly noticeable in the social sciences, such as accounting, social welfare, and economics/business administration. Second, papers written by scholars from Seoul National University, Yonsei University, and Korea University were often published in high-impact factor journals. Among those articles, the rate of professor-student co-authorship increased by $21.6 \%$ for 7 years. Third, the increase in professor-student co-authored articles published in high- impact factor journals was even sharper. These findings indicate that perceptions of professorstudent co-authorship have changed in the humanities and social sciences. In the near future, positive perceptions toward joint research and joint authorship between professors and students are expected to become more widespread.

Received: January 29, 2017

Accepted: February 10, 2017

Correspondence to Eun Seong Hwang eshwang@uos.ac.kr

ORCID

Eun Seong Hwang

http://orcid.org/0000-0001-8580-8444
Keywords

Authorship; Bibliometrics; Humanities; Republic of Korea; Social sciences 


\section{Introduction}

In science and engineering, single-authored papers are rare, and co-authored papers are common. Joint research with coauthorship is in general superior in terms of scope and quality compared to individual research and single-authored papers, and thus, has the advantage of leading to results with greater influence. Additionally, by going through the process of discussion with other authors, research process can be better monitored and results can be better analyzed. Since distortions in results analysis and presentation can also be better excluded through this process, co-authorship is a desirable study method in the sense that it helps guarantee research integrity. Meanwhile, a student under the guidance of a professor should be recognized as a co-author in a paper if the student collected research materials and data that were used in the write-up of the paper. In many cases, though, professors complete the final version of a paper by improving the logic of what the student writes as a first draft. The process of producing the final version requires the professor to play the role of exploring and including novel research ideas, which is not an easy task. Through all these processes, the supervising professor and the student become not only partners but co-owners, or in other words, co-authors of the article. The responsibility for the content of the paper rests upon both co-authors as well.

However, many academic areas in the humanities and social science have only a small number of professor-student coauthored papers compared to the number of papers co-authored by professors and/or researchers. Accordingly, improvements must be made in the awareness of joint research culminating in professor-student co-authorship. In this study, we researched changes in the number of articles co-authored between professors and students between 2 phases-2004 to 2006 (3 years) and 2011 to 2013 (3 years) — and used articles from humanities and social science journals as resources. The reason for excluding the natural sciences and engineering fields in this paper is the stark difference in the co-authorship rate between the social sciences (12\%) and the natural sciences and engineering (93\%), which has been discussed in previous research [1]. While joint research and joint authorship are generally accepted in the natural sciences and engineering, they are not commonly found in the humanities and social sciences. This study, hence, aimed to assess the current state of co-authorship between professors and students in the humanities and social sciences.

\section{Methods}

The academic journals analyzed in this study were the 36 highest-ranked journals by Korean Citation Index (KCI) impact factor (IF) (search criteria: IF, impact factor; 'as of 2012', 'five-year period') and 20 lower-ranked journals, drawn from the humanities and social sciences journals indexed in the KCI (Suppl. 1). The lower-ranked journals were chosen based on having similar fields and similar numbers of published articles to the selected high-ranked IF journals; therefore, they do not comprise the 20 journals with the absolute lowest IFs. Following the classification of the KCI, these journals were categorized into 13 research areas and were analyzed accordingly: accounting, social welfare, economics/business administration, sociology/social sciences, administrative science, political science and diplomacy, education, law, policy science, regional development, history, linguistics, and Korean and Korean literature. In this study, research areas in which surveys and experimental approaches are commonly used were included. The fields of the humanities-in particular, literature, history, and philosophy-were scarcely included. For this study, 10,930 articles from 56 journals during the 2 phases-2004 to 2006 (phase 1, 3-year period) and 2011 to 2013 (phase 2, 3-year period)-were chosen. Among those articles, there were 4,820 co-authored articles, and the articles were divided into professor-student co-authorship and other types of co-authorship. Moreover, articles were classified by the institutional affiliation of the authors. Articles with corresponding authors from Seoul National University, Korea University, and Yonsei University were considered to be 'SKY university articles' (1,123 papers), and other articles were considered to be 'non-SKY university articles' (3,697 papers). The number of professor-student co-authored articles was counted in each group. Likewise, among the articles published in high-IF and low-IF journals (7,772 articles from 36 high-ranked journals and 3,158 articles from 20 low-ranked journals), the number of professor-student co-authored papers was aggregated and compared. In most cases, whether the article was co-authored was checked by searching students' dissertations. In the cases of search failures, including cases of incomplete dissertations, inquiries were made by phone to college departments. If the student was already working as a full-time instructor or as a professor in another university at the time of publication, then the co-authorship was not considered to be between a supervising professor and student, but between a professor and a professor, and such articles were therefore excluded from the count of articles co-authored by professors and students.

\section{Results}

Changes in professor-student co-authored journal articles in the humanities and social sciences

Among the 56 journals in 13 research areas of the humanities 
and social sciences, we examined the co-authorship of articles published during the first and second phases in the journals with the highest IFs in each field. We categorized co-authorship as professor-student co-authorship if an article was coauthored by a professor and student from the same department, while other co-authored articles were considered to be examples of general co-authorship, and we investigated changes in co-authorship over the period of our study.

Between the first and second phase, the total proportion of co-authored articles increased by 15.9 percentage points, from $34.7 \%$ to $50.6 \%$. The percentage of articles with general coauthorship increased from $34.8 \%$ to $47.4 \%$, and the percentage of professor-student co-authored papers increased from $9.9 \%$ to $20.7 \%$. In most academic fields, the ratio of both general co-authorship and professor-student co-authorship increased similarly in the second phase (Fig. 1). Co-authorship was already widespread in the fields of accounting, social welfare, economics, business administration, education, policy studies, and regional development, with a co-authorship rate of more than $50 \%$ of articles. Within the 10 -year period encompassed by our study, the ratio increased even more, and in the case of accounting, general co-authorship accounted for $85 \%$ of all articles and professor-student co-authorship accounted for $44 \%$. In contrast, in the field of law and history, the ratio of co-authorship was very low in the first phase, and even in the second phase, the 2 types of collaboration were only present in $3.5 \%$ and $1.8 \%$ of articles, respectively. In history, among the investigated articles (57 from the first phase and 45 from the second phase) from a Korean archaeological journal, the number of articles with general co-authorship decreased from 7 to 6 and number of professor-student co-authored articles decreased from 3 to 1 . It is difficult to consider this a representative result due to the small sample size. However, the increase in professor-student co-authorship in comparison with the increase in general co-authorship did not reach statistical significance. The relative increase of profes-

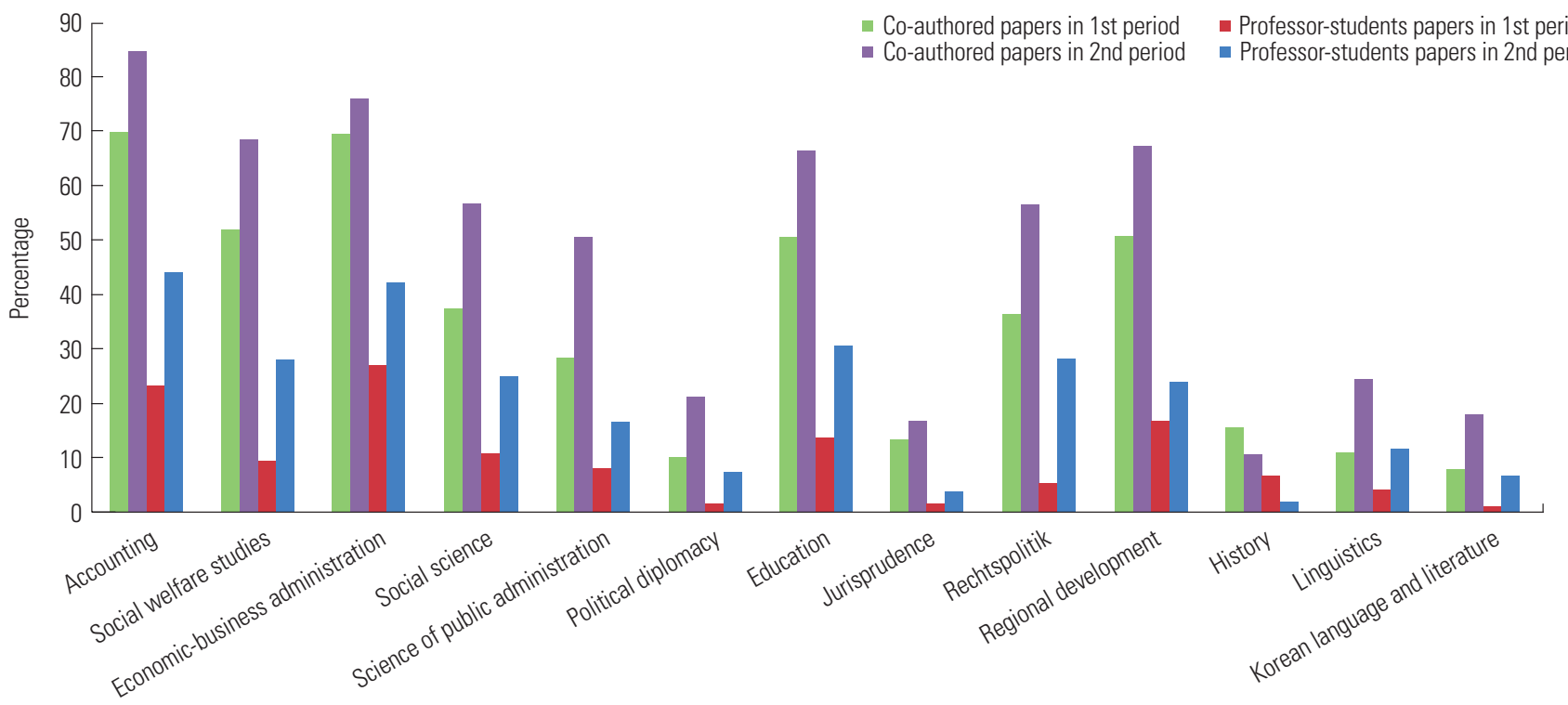

Fig. 1. Percentage of co-authored papers in different academic fields during periods 1 and 2 of the study.

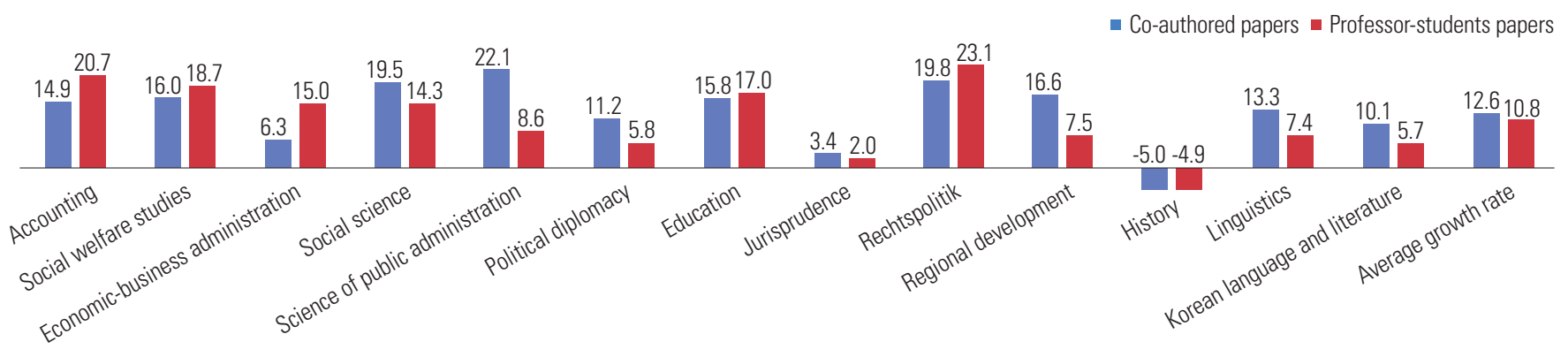

Fig. 2. Changes in the percentage of co-authored papers during periods 1 and 2 of the study. 
sor-student co-authorship was the largest in economics and business administration, with an increase of 2.4 times, but there were also cases where the increase was by less than 1 article (Fig. 2). However, this result does not weaken the significance of the general increase in co-authorship.

\section{Ratio of student-professor co-authored articles by} researchers at Seoul National University, Korea University, and Yonsei University

Next, we attempted to determine how the change in the ratio of professor-student co-authorship was related to excellence in research and research systems. First, we examined changes in the ratio of professor-student co-authorship among articles with corresponding authors from Seoul National University, Korea University, and Yonsei University (SKY articles) and other articles (non-SKY articles). The reason for comparing these 2 groups was not based on the assumption that the human resources of Seoul National University, Korea University, and Yonsei University and their students are superior, but instead on the assumption that these universities may have a higher graduate student ratio and more research funding, and therefore be characterized by research excellence stemming from this research system. This is partly supported by the following facts. In all of the investigated humanities and social science fields, more articles were published in the 36 high-IF journals during the second phase by professors from these 3 universities than by the other group. For example, in account- ing, 32\% of the total 396 papers (127 papers) were published by professors from these 3 universities. In addition, in 19 journals, corresponding more than half of the 36 high-ranked journals, more than $10 \%$ of the articles were by professors from these 3 universities, with an average share exceeding $30 \%$. In contrast, the proportion of SKY articles in $80 \%$ of the 20 low-IF journals (16 journals) did not exceed 10\% (Table 1).

Our analysis showed that the share of SKY articles across the humanities and social sciences somewhat decreased in all journals (by $2.0 \%$ in the high-IF journals and $1.1 \%$ in the lowIF journals). However, the ratio of professor-student co-authorship in the SKY articles increased by 21.6 percentage points (Fig. 3). In the non-SKY articles, the ratio of professorstudent co-authorship increased, but the increase was relatively low, at 9.5 percentage points. In most fields the ratio of professor-student co-authorship in SKY articles increased by more than 20 percentage points (more than 30 percentage points in social welfare, economics, business administration, education, policy studies, regional development, and linguistics), but the ratio in non-SKY articles increased by less than 20 percentage points (Fig. 3).

Changes in the ratio of co-authorship in the high-IF and low-IF journals

Next, we compared trends in professor-student co-authorship in the 36 high-IF journals and the 20 low-IF journals. We need to add a few words to avoid misunderstandings that may

Table 1. Changes in the percentage of the papers co-authored in general, by professors and students, and by SKY professors and students

\begin{tabular}{|c|c|c|c|c|}
\hline Journals according to $\mathrm{KCI} I \mathrm{~F}$ & $\begin{array}{l}\text { Increase in the number of } \\
\text { co-authored papers (general) }\end{array}$ & $\begin{array}{l}\text { Increase in the number of } \\
\text { papers co-authored by } \\
\text { professors and students }\end{array}$ & $\begin{array}{l}\text { Increase in the number } \\
\text { of SKY papers }\end{array}$ & $\begin{array}{l}\text { Increase in the number of } \\
\text { papers co-authored by SKY } \\
\text { professors and students }\end{array}$ \\
\hline Top 36 journals & $15.9 \%$ & $13.0 \%$ & $-2.0 \%$ & $21.6 \%$ \\
\hline Lower 20 journals & $22.2 \%$ & $11.5 \%$ & $-1.1 \%$ & $19.8 \%$ \\
\hline
\end{tabular}

SKY, Seoul National University, Korea University, and Yonsei University; KCl, Korean Citation Index; IF, impact factor.

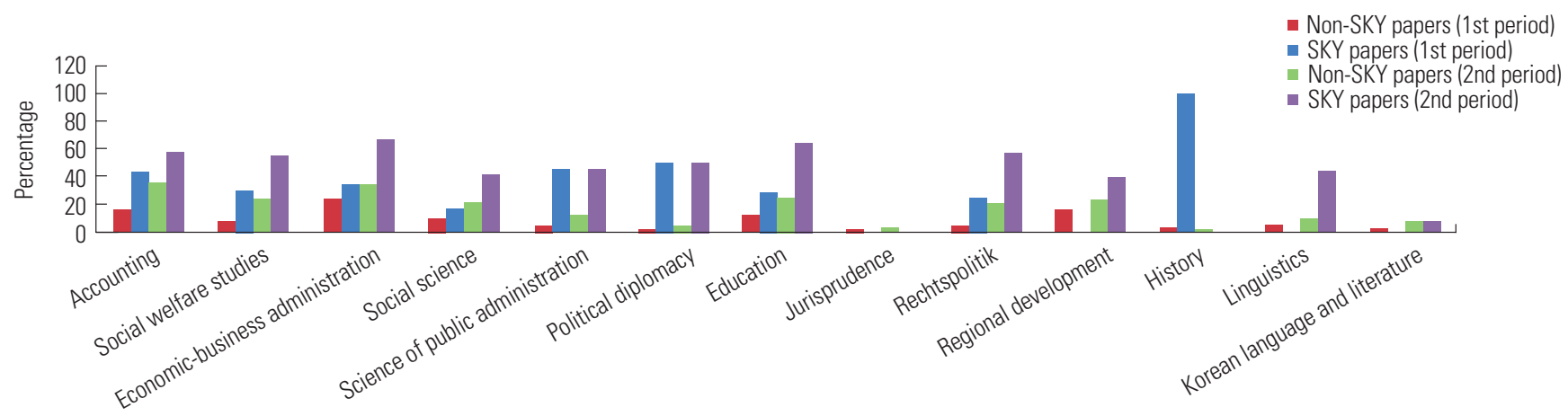

Fig. 3. Percentage of professor-student co-authored papers from researchers at Seoul National University, Korea University, and Yonsei University (SKY papers) and non-SKY papers. 


\section{science editing /}

arise about the selection criteria. First, although a high IF does not always indicate a better academic journal, it can be used as an effective index to filter academic journals above a certain level, and therefore it is possible to use IF to distinguish higher-ranked and lower-ranked groups of journals. The high-IF journals had a KCI IF of 2.00 on average, and the low-IF journals had an average KCI IF of 0.16 , indicating a considerable difference in the level of domestic journals indexed in the KCI (Table 1).

Co-authorship in high-IF journals increased by 15.9 percentage points, and by 22.2 percentage points in low-IF journals. This suggests that the proportion of co-authorship in the low-IF journals was very low, but has recently become more similar to the high-ranked journals. However, professor-student co-authorship increased by 13 percentage points in the high-IF journals and 11.5 percentage points in the low-IF journals. As such, a greater increase was observed in the rate of professor-student co-authorship than in the rate of general co-authorship in high-IF journals. In addition, professor-student co-authorship in the SKY articles increased by 21.6 percentage points in high-IF journals and by 19.8 percentage points in low-IF journals (Table 1). This also shows a positive correlation between the excellence of the research and the tendency for professor-student co-authorship.

\section{Discussion}

In the humanities and social sciences, a significant change has occurred in the ratio of professor-student co-authorship, with an increase of more than 10 percentage points over the 10year period from 2004 to 2013 (Figs. 1, 2). This change can be attributed to greater collaboration between existing researchers and new generations of researchers, and an increase in the number of graduate students.

SKY articles tended to be published in comparatively higher-IF journals indexed in the KCI. This suggests that their articles may have a relatively higher citation index or influence, or in other words, academic excellence (Table 1). In addition to the academic excellence of the research, the excellence of the research system suggests that the research guidance of graduate students at these universities is better managed.

In most fields, the ratio of professor-student co-authorship in SKY articles increased by more than 20 percentage points, but the ratio in non-SKY articles increased by less than 20 percentage points, indicating that this system of co-authorship significantly increased in articles from researchers at universities exhibiting academic excellence in research or the research system. This demonstrates that if the research and research management are excellent, students tend to be recognized as co-researchers.
This study focused on the academic fields of the humanities and social studies, but the result is that we have started to perceive the academic practice of students in the academy as collaborative research rather than merely a process of training. In addition, these results demonstrate the emergence of positive perceptions of professor and student co-authorship. Additionally, the fact that this phenomenon did not occur in only 1 or 2 fields shows that the gap between concepts and perceptions of authorship is narrowing in all academic fields in Korea. This suggests the hopeful message that it may be possible to set common standards for all ethical issues encountered in research in all academic fields.

The results of this study have another important message from a practical point of view. There is a misperception about collaborative research between professors and students in our society. In particular, court rulings have prohibited the acknowledgment of professor-student co-authorship [2]. In September 2004, the Seoul Administrative Court issued a ruling that "it is wrong for a professor to claim co-authorship when a student's dissertation is published." The following is a part of the 2004 judgment [2].

"The plaintiff, as an advisor to A, has made substantial contributions beyond the scope of guidance as an advisor in the research process and preparation of the doctoral dissertation, so the work (journal article) is claimed to be a co-creation of plaintiff and A" (...) "If the plaintiff played a leading role in the process of research and the dissertation of the student as the plaintiff claimed, or, if they have reached a level of collaborative research that is beyond mere instruction, considering that the academic maturity of the plaintiff is higher than that of $\mathrm{A}$, the dissertation written during such a process should be regarded as the work of the plaintiff, rather than being a dissertation of A. In this case, A has used the results of the plaintiff's research in his doctoral dissertation, therefore not only leaving a room to doubt the authenticity of the value of the degree, but also raising another criticism that the plaintiff has failed to fulfill the role of a thesis advisor or thesis review chairperson." (...) "Even if the practice of co-publishing with the advisor is common, this is a distorted phenomenon in the academic world, and therefore it cannot be the research of the plaintiff."

In this case, it is unknown whether substantial research guidance was provided that could be considered joint research between the professor and the student. However, this ruling contains a serious error due to the ignorance of judges regarding the general process of academic research activities. In particular, the ruling referred to the practice of collaborative research and co-authorship between professors and students, which has existed for many years in academia, as a "distorted phenomenon." The results of this study document 
the increasing proportion of professor-student co-authorship in scholarship, and indicate that there is a major gap between research practices in academia and this judgment. Based on the results of this study, the Korean academy should continue to encourage collaborative research between professors and students in all fields, including the fields of literature, history, and philosophy.

In conclusion, these results show that in the humanities and social sciences in Korea, the ratio of professor-student coauthorship has increased in almost all fields. Additionally, we can see that the academic practice of students is recognized as collaborative research rather than merely a training process, and that professor-student co-authorship is finding its place in the humanities and social sciences in Korea.

\section{Conflict of Interest}

No potential conflict of interest relevant to this article was reported.

\section{Supplementary Material}

Suppl. 1. Categories and Korean Citation Index impact factors of the journals studied

\section{References}

1. Lee SH. Collaboration in the field of social vs. natural science. Paper presented at: the first conference of the Korean Society for Information Management; 1994 Dec; Seoul, Korea. p. 47-50.

2. Judicial Precedent of Seoul Administrative Court. 2004 September. 2003Guhap6498. 